\title{
Avaliação isocinética em atletas paraolímpicos
}

\author{
Antônio Carlos Silva ${ }^{1}$ e Marília dos Santos Andrade ${ }^{2}$
}

\section{RESUMO}

O equilíbrio dos parâmetros de força muscular nas articulações é de grande relevância, tanto no aspecto clínico como para o desempenho atlético. Informações sobre os valores desses parâmetros em atletas olímpicos e principalmente nos paraolímpicos são raras. Neste trabalho apresentamos os resultados da avaliação muscular isocinética (Cybex 6000) realizada na equipe brasileira que participou dos Jogos Paraolímpicos de Sidney 2000. Foram avaliados os músculos flexores e extensores dos joelhos de 11 jogadores de futebol (paralisia cerebral, idade 24,6 $\pm 4,8$ anos; peso $67 \pm 5,5 \mathrm{~kg}$; altura $177,7 \pm 3,8 \mathrm{~cm})$ e 12 de basquetebol (deficientes mentais, idade $24,7 \pm 4,4$ anos; peso 76,6 $\pm 13,4 \mathrm{~kg}$; altura $184,4 \pm 10 \mathrm{~cm}$ ), e os músculos rotadores internos e rotadores externos de ombros de seis judocas (deficientes visuais, idade 29,8 $\pm 5,6$ anos; peso $87 \pm$ $21,6 \mathrm{~kg}$; altura $171,5 \pm 6,9 \mathrm{~cm})$. Os jogadores de futebol apresentaram relação de equilíbrio muscular entre flexores e extensores de joelhos dentro dos parâmetros de normalidade apesar da fraqueza muscular imposta pela paralisia cerebral. Nos judocas o equilíbrio muscular entre rotadores externos e rotadores internos mostrou-se dentro dos limites de normalidade. A principal característica dos jogadores de basquetebol foi o valor de pico de torque próximo do esperado para indivíduos hígidos não atletas. Os valores numéricos estão apresentados no texto para serem usados como referência para profissionais da área.

Palavras-chave: Testes anaeróbios. Força anaeróbia. Atletas paraolímpicos.

1. Doutor em Ciências da Universidade Federal de São Paulo, Escola Paulista de Medicina, Unifesp-EPM.

2. Doutoranda em Reabilitação pela Unifesp-EPM. Especialista em Aparelho Locomotor no Esporte. Especialista em Fisiologia do Exercício pelo Centro de Estudos de Fisiologia do Exercício, CEFE-Unifesp-EPM.

Submetido em: 4/4/02

Versão revisada recebida em: 1/5/02

Aceito em: 19/5/02

Endereço para correspondência:

CEFE-Centro de Estudos de Fisiologia do Exercício

Antônio Carlos Silva

Rua Botucatu, 862, 5o andar

04023-062 - São Paulo, SP

E-mail: anton@ecb.epm.br

\section{ABSTRACT}

\section{Isokinetic evaluation in paralympic athletes}

The balance between agonist and antagonist muscles in the joints is of great relevance to clinical practice and to sports performance. Information about these parameters amongst Olympic and especially Paralympic athletes is scarce. The purpose of this study was to present the results of the isokinetic evaluation (Cybex 6000) of the Brazilian team that participated in the Sydney Paralympic Games. The flexor and extensor muscles of the knee were evaluated in 11 soccer players with cerebral palsy (age: $24.6 \pm$ 4.8 years; weight: $67 \pm 5.5 \mathrm{~kg}$; height: $177.7 \pm 3.8 \mathrm{~cm})$ and in 12 basketball athletes with mental disability (age: 24.7 years; weight: $76.6 \mathrm{~kg}$; height: $184.4 \pm 10 \mathrm{~cm})$; for the judo blind athletes (age: $29.8 \pm 5.6$ years; weight: 87 $\pm 21.6 \mathrm{~kg}$; height: $171.5 \pm 6.9 \mathrm{~cm}$ ), the shoulder rotator muscles were tested. Findings show that the soccer players have a normal hamstring: quadriceps strength ratio despite the muscle weakness imposed by cerebral palsy. For the judo athletes, the ratio between external and internal rotators of the shoulder was within the normal range. The results for peak torque in the basketball players were similar to those of non-athletic individuals without disabilities. The values are presented in the article in order to be used as reference for the professionals involved in the area of disabled sports.

Key words: Anaerobic tests. Anaerobic power. Paralympic athletes.

\section{INTRODUÇÃO}

A deficiência da força muscular influencia negativamente a estabilidade $\operatorname{articular}^{1}$ e o desempenho atlético ${ }^{2-6}$. Desta forma, uma avaliação precisa da função muscular tem sido de fundamental interesse clínico, principalmente quando o objetivo é prevenir lesões através da identificação precoce de deficiência contralateral de um grupo muscular, ou de desequilíbrio entre músculos antagonistas de uma articulação.

Existem algumas formas de avaliar a força muscular. Dinamômetros de cabo podem medir a força isométrica, pesos livres ou equipamentos isotônicos podem medir a força de uma repetição máxima (1-RM) e mais recentemente os dinamômetros isocinéticos podem quantificar precisa- 
mente o desempenho muscular, assim como oferecer valores da comparação contralateral do mesmo grupo muscular, ou comparação homolateral de grupos musculares antagonistas. Portanto, este tipo de avaliação pode fornecer informações importantes que podem ser usadas para melhorar a performance ${ }^{7-9}$ ou para identificar fraquezas musculares que predispõe o atleta a lesão músculo-esqueléti$\mathrm{ca}^{10}$.

Existe, porém, uma escassez de valores normativos da capacidade muscular isocinética de indivíduos saudáveis. Para os atletas portadores de deficiência física a escassez é ainda maior.

Desta forma, o objetivo deste estudo foi disponibilizar os dados obtidos na avaliação muscular isocinética dos atletas brasileiros que participaram dos Jogos Paraolímpicos de Sidney 2000.

\section{METODOLOGIA}

Participaram do estudo 11 jogadores de futebol portadores de paralisia cerebral (idade: $24,6 \pm 4,8$ anos; peso: $67 \pm$ $5,5 \mathrm{~kg}$; altura: $177,7 \pm 3,8 \mathrm{~cm}), 12$ jogadores de basquetebol portadores de deficiência mental (idade: $24,7 \pm 4,4$ anos; peso: $76,6 \pm 13,4 \mathrm{~kg}$; altura: $184,4 \pm 10 \mathrm{~cm}$ ) e 6 judocas com deficiência visual (idade: $29,8 \pm 5,6$ anos; peso: $87 \pm$ $21,6 \mathrm{~kg}$; altura: $171,5 \pm 6,9 \mathrm{~cm}$ ).

Os jogadores de futebol e de basquetebol foram submetidos a uma avaliação de flexores e de extensores do joelho. Os judocas fizeram avaliação de rotadores internos e externos de ombro. Para tanto, utilizamos um dinamômetro isocinético Cybex 6000 (Ronkonkoma, NY). Os indivíduos realizaram um aquecimento de oito minutos pedalando com 25 watts em uma bicicleta estacionária da marca Cybex. O membro dominante foi avaliado primeiro. O protocolo de teste constou de uma série de cinco repetições a $60 \%$ s para avaliar a força (pico de toque) que foi precedida por 3 repetições para familiarização do voluntário com o equipamento. O protocolo de avaliação dos membros inferiores foi igual ao dos membros superiores.

\section{RESULTADOS}

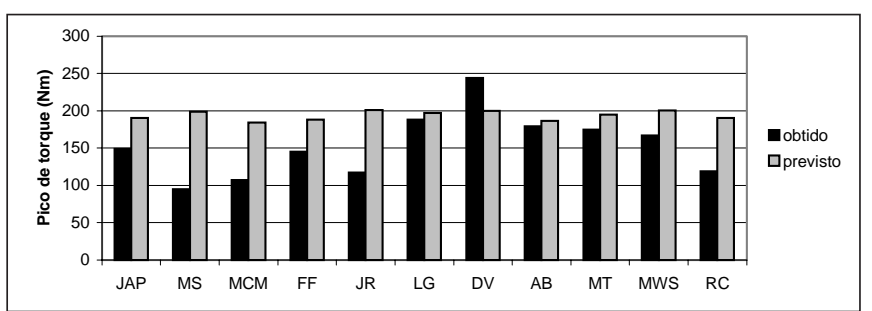

Fig. 1 - Pico de torque obtido e previsto dos extensores do joelho da perna não-dominante dos jogadores de futebol

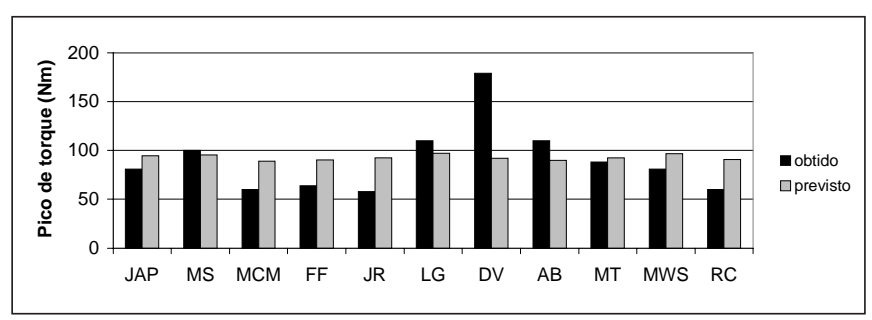

Fig. 2 - Pico de torque obtido e previsto dos flexores do joelho da perna não-dominante dos jogadores de futebol

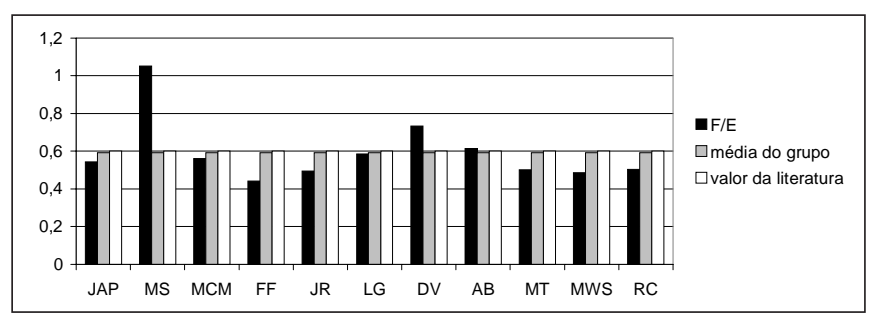

Fig. 3 - Relação entre pico de torque dos flexores e extensores $(F / E)$ de joelho da perna não-dominante

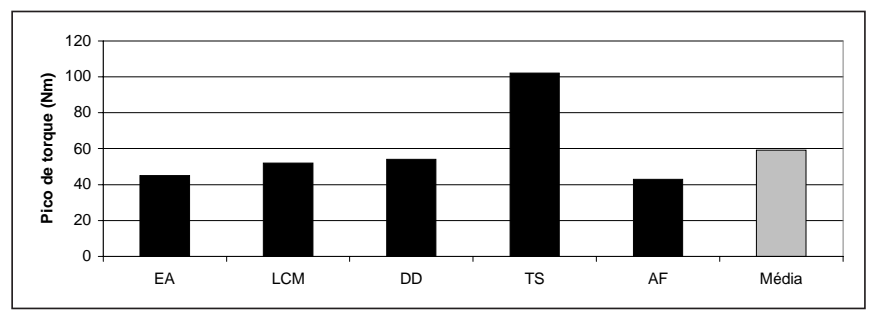

Fig. 4 - Pico de torque obtido dos rotadores internos de ombro dominante dos lutadores de judô

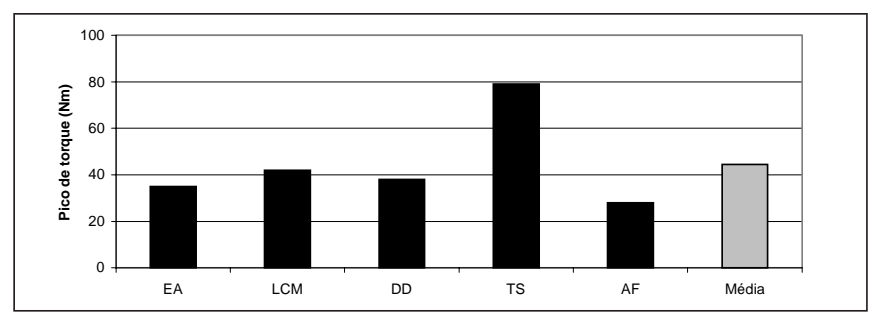

Fig. 5 - Pico de torque obtido dos rotadores externos de ombro dominante dos lutadores de judô

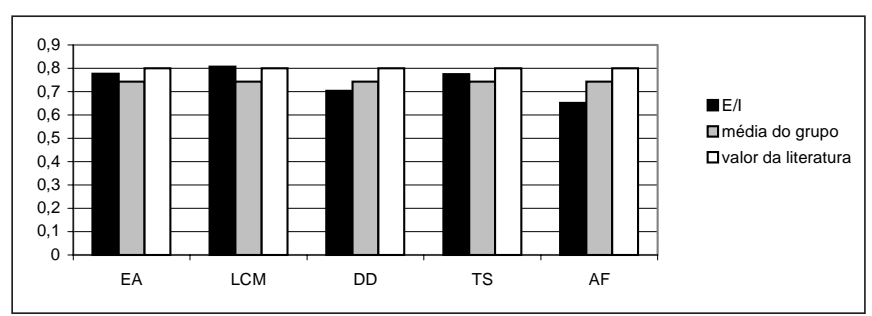

Fig. 6 - Relação entre rotadores externos e internos (E/I) do ombro dominante de lutadores de judô 


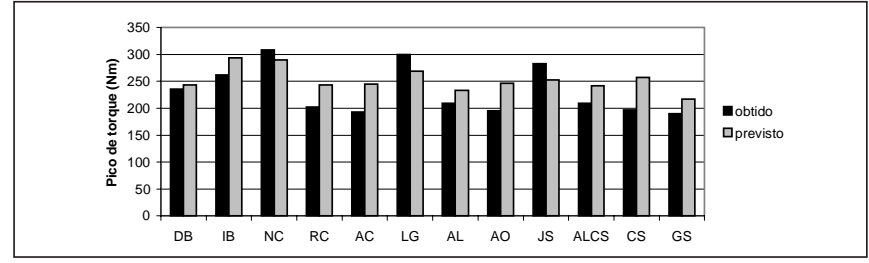

Fig. 7 - Pico de torque obtido e previsto dos extensores do joelho da perna dominante dos jogadores de basquete

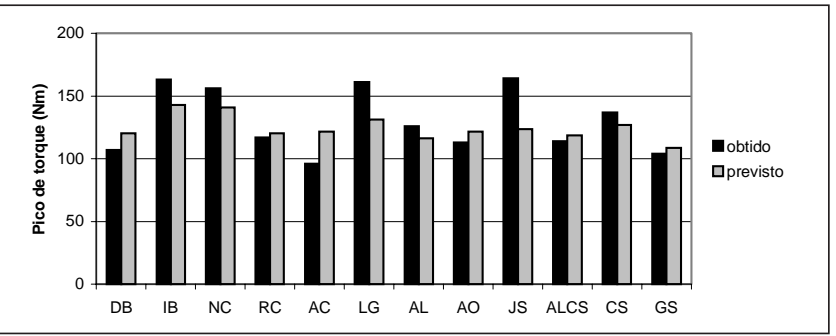

Fig. 8 -Pico de torque obtido e previsto dos flexores do joelho da perna dominante dos jogadores de basquete

\section{DISCUSSÃO}

Observamos que os jogadores de futebol, que eram todos paralisados cerebrais, e que em sua maioria apresentavam hemiparesia espástica, demonstraram valores de pico de torque mais baixos do que os valores previstos para indivíduos sem deficiência física de mesmo peso, altura, idade, sexo e nível de atividade física ${ }^{11}$ (gráficos 1 e 2). Porém, eles mantiveram o equilíbrio muscular entre flexores e extensores do joelho em valores normais $(50-60 \%)^{7,12-14}$ (gráfico 3). Desta forma, apesar destes indivíduos apresentarem uma fraqueza muscular, o que já era previsto com base nos dados de literatura ${ }^{10,15}$ esta fraqueza não é desproporcional entre flexores e extensores do joelho.

No judô observamos que o atleta T.S. (95kg) é um pouco mais pesado do que a média do seu grupo $(87 \mathrm{~kg})$. Mesmo considerando este fato, observamos que o atleta é muito mais forte do que os outros judocas (gráficos 4 e 5), e ele foi o único atleta que ganhou uma medalha dos jogos paraolímpicos de Sidney. O que corrobora a idéia de que a força muscular e a performance estão correlacionadas.

$\mathrm{O}$ equilíbrio muscular entre rotadores internos e externos destes atletas está muito próximo da normalidade (70$80 \%)^{16-18}$ (gráfico 6). Isto é muito importante para estes atletas porque na prática esportiva do judô uma tensão muito grande é exercida nesta articulação e, para que não ocorra prejuízo articular é importante que estes músculos estejam aptos para absorver tais tensões.

Nos jogadores de basquetebol observamos que os valores obtidos por estes atletas estão muitos próximos dos valores previstos para não deficientes (gráficos 7 e 8), sugerindo que a deficiência mental destes indivíduos exerce pouca ou nenhuma influência no desenvolvimento de força muscular para atletas desta modalidade esportiva e com este nível de comprometimento.

\section{AGRADECIMENTOS}

- Comitê Paraolímpico Brasileiro (CPB)

- Secretaria Nacional de Esportes

- Rede Cenesp/Unifesp

- Associação Fundo de Incentivo à Psicofarmacologia (Afip)

- Universidade Federal de São Paulo (Unifesp)

\section{REFERÊNCIAS}

1. Shirakura K, Kato K, Udagawa E. Characteristics of the isokinetic performance of patients with injured cruciate ligaments. Am J Sports Med 1992;20: 754-60.

2. Noyes FR, Barber SD, Mangine RE, Abnormal lower limb symmetry determined by function hop tests after anterior cruciate ligament rupture. Am J Sports Med 1991;19:513-8.

3. Sachs RA, Daniel DM, Stone ML, Garfein RF. Patellofemural problems after anterior cruciate ligament reconstruction. Am J Sports Med 1989;17:760-5.

4. Tegner Y, Lysholm J, Lysholm M, et.al: Strengthening exercises for ald cruciate ligament tears. Acta Orthop Scand 1986;57:130-4.

5. Wiklander J, and Lysholm J. Simple tests for surveying muscle strength and muscle stiffness in sportsmen. International Journal Sports Medicine 1987;8:50-4.

6. Wilk KE, Andrews JR. Current concepts in the tretment of anterior cruciate ligament disruption. J Orthop Sports Phys Ther 1992;15:279-91.

7. Chan KM, Maffulli N. Principles and practice isokinetics in sports medicine and rehabilitation. Hong Kong: Williams \& Wilkins, 1996.

8. Dvir Z. Isokinetics, muscle testing, interpretation and clinical applications. New York: Churchill Livingstone, 1995.

9. Perrin D. Isokinetic exercise and assessment, Champain, IL: Human Kinetics, 1993;153-158.

10. Brown A. Review: Physical fitness and cerebral palsy. Child: Care, Health and Development 1975;1:43-52.

11. Neder JA, Nery LE, Shinzato GT, Andrade MS, Peres C, Silva AC. Reference values for concentric knee isokinetic strength and power in nonathletic men and women from 20 to 80 years old. J Orthop Sports Phys Ther 1999;29: 116-26.

12. Brown LE. Isokinetics in human performance. Champain, IL. Human Kinetics, 2000.

13. Damiano D, Kelly L, Vaughn CL. Effects of quadriceps femoris muscle strengthening on crouch gait in children with spastic diplegia. Phys Ther 1995;75:658-67.

14. Holmes JR, Alderink GJ. Isokinetic strength characteristics of the quadríceps femoris and hamstrings muscles in high school students. Physical Therapy 1984;64:914-8.

15. Ann Mac Phail HE. Effect of isokinetic strength-training on functional ability and walking efficiency in adolescents with cerebral palsy. Developmental Medicine and Child Neurology 1995;37:763-75.

16. Codine P, Bernard PL, Pocholle M, Benaim C, Brun V. Influence of sports discipline on Shoulder Rotator cuff Balance. Med Sci Sports Exerc 1997;29: 1400-5.

17. Leroux JL, Codine P, Thomas E, Pocholle M, Mailhe D, Blotman F. Isokinetic Evaluation of rotational strength in normal shoulders and shoulders with impingement syndrome. Clinical Orthopaedics and Related Research 1994;304:108-15

18. Rupp S, Berninger K, Hopf T. Shoulder problems in high level swimmersimpingement, anterior instability, muscular Imbalance? Orthopedics and Clinical Science 1995;16:557-62. 